\title{
K2 Reveals Pulsed Accretion Driven by the 2 Myr Old Hot Jupiter CI Tau b
}

\author{
Lauren I. Biddle ${ }^{1,2}$ (D), Christopher M. Johns-Krull ${ }^{3}$, Joe Llama ${ }^{1}\left(\mathbb{D}\right.$, Lisa Prato $^{1}$, and Brian A. Skiff ${ }^{1}$ \\ ${ }^{1}$ Lowell Observatory, 1400 W. Mars Hill Road, Flagstaff, AZ 86001, USA; lbiddle@lowell.edu \\ ${ }^{2}$ Department of Physics \& Astronomy, Northern Arizona University, 527 S. Beaver Street, Flagstaff, AZ 86011, USA \\ ${ }^{3}$ Department of Physics \& Astronomy, Rice University, 6100 Main Street, MS-108, Houston, TX 77005, USA \\ Received 2017 November 10; revised 2018 January 13; accepted 2018 January 17; published 2018 February 2
}

\begin{abstract}
CI Tau is a young ( $\sim 2$ Myr) classical T Tauri star located in the Taurus star-forming region. Radial velocity observations indicate it hosts a Jupiter-sized planet with an orbital period of approximately 9 days. In this work, we analyze time series of CI Tau's photometric variability as seen by $K 2$. The light curve reveals the stellar rotation period to be $\sim 6.6$ days. Although there is no evidence that CI Tau b transits the host star, a $\sim 9$ day signature is also present in the light curve. We believe this is most likely caused by planet-disk interactions that perturb the accretion flow onto the star, resulting in a periodic modulation of the brightness with the $\sim 9$ day period of the planet's orbit.
\end{abstract}

Key words: planet-disk interactions - stars: activity - stars: individual (CI Tau) - stars: magnetic field

\section{Introduction}

Detecting and characterizing exoplanets around very young stars enables a direct view into the formation and evolution of planetary systems. Mature planetary systems encompass a range of configurations including hot Jupiters orbiting within tenths of an au from their host stars and super-Earths orbiting within the habitable zone of low-mass stars (Howard et al. 2010; Borucki et al. 2011; Kopparapu 2013; Burke et al. 2014; Winn \& Fabrycky 2015). While there are now nearly 3000 confirmed exoplanets ${ }^{4}$ around main-sequence stars, post-main-sequence stars, brown dwarfs, and pulsars, only a handful of candidates have been found around the youngest of stars. One reason for the lack of detected planets around young stars is simply that there are very few nearby star-forming regions, with most being beyond $120 \mathrm{pc}$. Consequently, radial velocity (RV) measurements become difficult because of the faintness of the host stars. Even for bright young stars, exoplanet detection remains difficult in relatively nearby star-forming regions such as Ophiuchus and Taurus. Young classical $\mathrm{T}$ Tauri stars typically have optically thick, actively accreting circumstellar disks and exhibit stellar activity signatures caused by cold starspots as well as variable accretion of disk material channeled onto the star along strong magnetic field lines (Herbst et al. 2007). These astrophysical phenomena manifest themselves in both photometric and RV variability studies, making exoplanet detection around young stars extremely challenging (Queloz et al. 2001; Bouvier et al. 2007; Robertson et al. 2014).

Despite these challenges, there have been a number of recently announced exoplanets around $\mathrm{T}$ Tauri stars, detected through a variety of methods, including V830 Tau b (Donati et al. 2016), K2-33 b (Mann et al. 2016; David et al. 2016), and TAP 26 b (Yu et al. 2017). Our team reported the RV detection of CI Tau b (Johns-Krull et al. 2016), a 2 Myr old hot Jupiter around a classical T Tauri star. CI Tau b was detected after an extensive radial velocity campaign combining both optical and infrared measurements in an effort to disentangle the planet signal from stellar induced RV signals. From the RV measurements, Johns-Krull et al. (2016) determined the

\footnotetext{
4 http://www.exoplanets.org (2018 January 12).
}

minimum mass of the planet to be $M_{p} \sin i=8.08 \pm$ $1.53 M_{\text {Jup }}$ and the orbital period to be $P_{\text {orb }}=8.9891 \pm$ 0.0202 days. Under the assumption that the stellar inclination is equal to that of the circumstellar disk $\left(i=45^{\circ} .7 \pm 11^{\circ} 1\right.$; Guilloteau et al. 2014), then $M_{p}=11.29 \pm 2.16 M_{\text {Jup. In }}$ addition, Johns-Krull et al. (2016) acquired ground-based photometry to probe CI Tau's photometric variability, which yielded a stellar rotation period of 7.1 days, though with considerable uncertainty.

A new opportunity to characterize CI Tau's photometric variability was provided by $K 2$, the extended mission of the Kepler Space Telescope (Borucki et al. 2010), intended to observe various fields in the ecliptic plane for periods of approximately 80 days (Howell et al. 2014). In this Letter, we present the $K 2$ photometry of the CI Tau system released on 2017 August 28, which provides further evidence for the presence of $\mathrm{CI} \mathrm{Tau} \mathrm{b}$, enabling us to disentangle the stellar rotation signal from that of the planet-disk interaction.

\section{Observations and Analysis}

K2 long-cadence time-series photometry of CI Tau (EPIC 247584113) was acquired during Campaign 13 between 2017 March 8 and May 27 UTC. We downloaded the light curve from the Milkulski Archive for Space Telescopes $\left(\mathrm{MAST}^{5}\right)$. We detrended the PDC_SAP (Jenkins et al. 2010) light curve with the K2SC pipeline (Aigrain et al. 2015, 2016), which uses Gaussian Process regression to remove instrumental systematics in the data while preserving astrophysical variability of the host star. The top left-hand panel of Figure 1 shows the PDC_SAP flux provided by the $K 2$ team and the postprocessing result from the $\mathrm{K} 2 \mathrm{SC}$ pipeline is shown in the bottom panel. Each light curve exhibits approximately $\sim 40 \%$ variability on multiple timescales within the 80 days of observations. The source of this variability is from surface inhomogeneities likely resulting from the combination of cool spots and accretion hot processes.

For each light curve, we computed a generalized LombScargle periodogram (Zechmeister \& Kürster 2009) using the

\footnotetext{
5 https://archive.stsci.edu/k2
} 

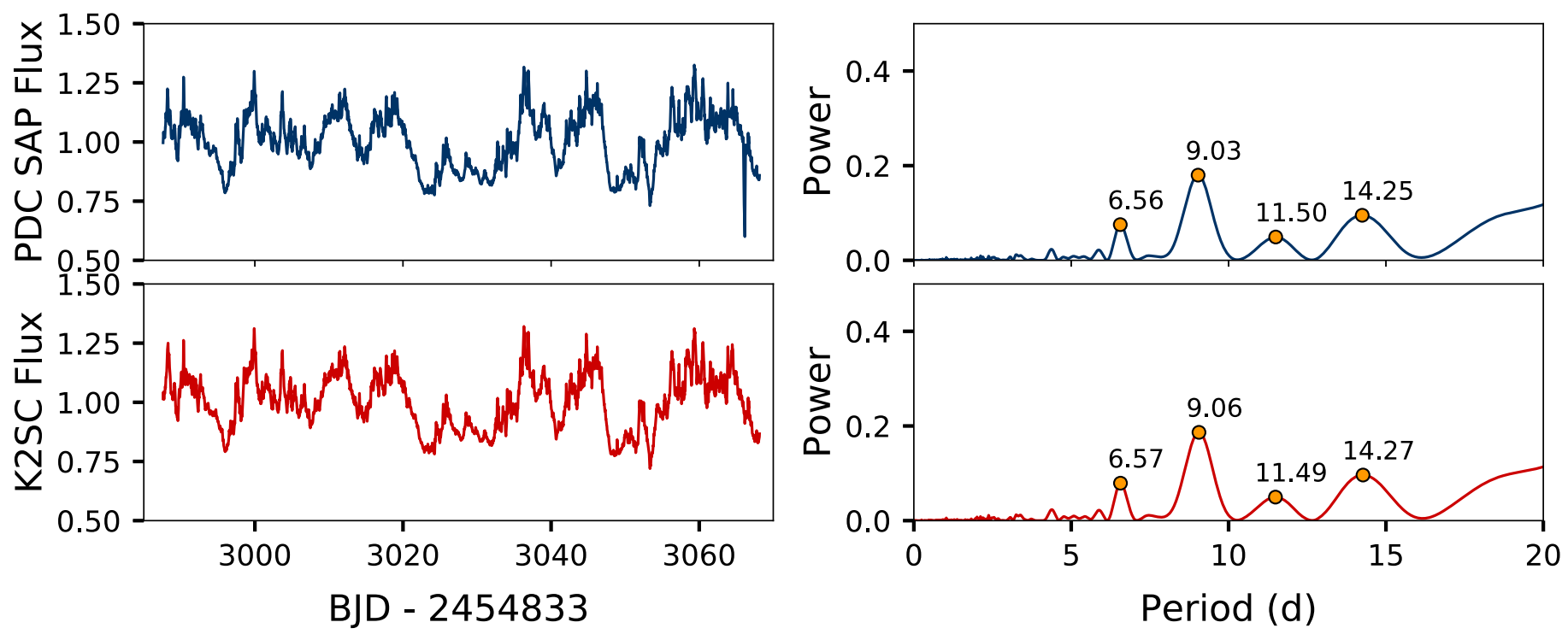

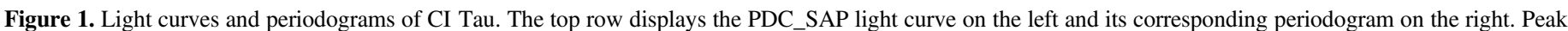
signals in the periodogram are marked with gold circles and their respective periods. The bottom row displays the same for the K2SC light curve.

PYTHON AstroML ${ }^{6}$ package. Each periodogram was computed between 0.042 and 80 days (consistent with the Nyquist sampling frequency; Press et al. 1992). We searched 10,000 points within this window to ensure that the peaks in the periodogram were well resolved. These limits and samplings are consistent with the recommendations of VanderPlas (2017). The periodograms show distinct signals at $6.56 \pm 0.22$ days and $9.03 \pm 0.51$ days for the PDC_SAP flux and at $6.57 \pm 0.24$ days and $9.06 \pm 0.50$ days for the K2SC flux (Figure 1, right). No strong peaks are seen at periods beyond 30 days; these are not shown. The period uncertainty was estimated from the FWHM of the periodogram power distribution surrounding each respective period (Ivezić et al. 2014). We calculated the false-alarm probability (FAP) using both the analytic solution (Zechmeister \& Kürster 2009) and a Monte Carlo bootstrap algorithm for both periods in each light curve. Both methods yield an FAP of $<10^{-6}$ for both periods. For completeness, we conducted identical analyses of detrended light curves from the EVEREST (Luger et al. 2016, 2017) and K2SFF pipelines (Vanderburg et al. 2015), and the results from each were consistent with both the PDC_SAP and K2SC.

To check whether the two periods hold throughout the series, we performed periodogram analyses on the first and second halves of the data sets separately (Figure 2). The $\sim 6.6$ day and $\sim 9.0$ day signals were present in both results. The locations of the $\sim 6.6$ day periods as well as their strength remained relatively constant compared to the $\sim 9.0$ day signal, which is clearly stronger during the first half of the observing period and exhibits slightly larger values ( $\sim 9.5$ days) compared to the full light curve ( $\sim 9.0$ days). We also calculated the FAP for the $\sim 6.6$ day and $\sim 9.0$ day periods for the first and second half light curves, results of which also returned $<10^{-6}$. The increased width of the peaks in the power spectrum at $\sim 6.6$ days and $\sim 9.0$ days when looking at the first or second half of the data is attributable to the lower frequency resolution that results from the decrease in the length of the time series when looking at only half of the data.

\footnotetext{
6 http://www.astroml.org/index.html
}

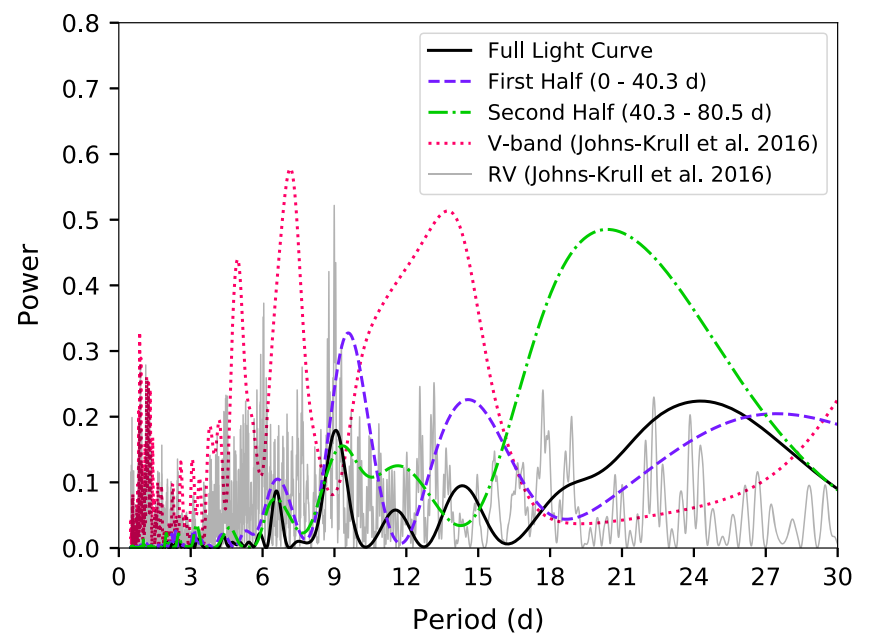

Figure 2. Direct comparison of the individual periodogram analyses of the full, first, and second halves of the K2SC light curve to an identical re-analysis of both the ground-based V-band photometry and combined visible and infrared RVs presented by Johns-Krull et al. (2016). The first half shows periodicity at $6.73 \pm 0.60$ days and $9.50 \pm 0.97$ days, while the second half shows at $6.62 \pm 0.51$ days and $9.41 \pm 0.95$ days. The PDC_SAP light curve yielded similar results $(6.81 \pm 0.60$ days and $9.47 \pm 0.96$ days for the first half and $6.50 \pm 0.47$ days and $9.41 \pm 0.96$ days for the second).

We constructed light curves phased to the $\sim 6.6$ day and $\sim 9.0$ day periods (Figure 3 ). The phased light curves appear to be coherent with both periodic signals. While there is considerable scatter in the phased plots, the points are concentrated in a way that appears periodic, which can be seen in the first and third rows of Figure 3. To better show this signal, we binned the photometric data points to 0.1 in phase, computed the mean and uncertainty of the mean within each bin, and then performed a sine fit to the binned data to measure the amplitude and significance of each signal. The fit to the \% 6.6 day phased light curves yielded an amplitude of $0.049 \pm 0.008$ normalized flux units for the PDC_SAP light curve (a $\sim 6 \sigma$ signal) and $0.050 \pm 0.007$ normalized flux units for the K2SC light curve (a $\sim 7 \sigma$ signal). The light curve sine wave amplitude of the $\sim 9.0$ day fit for each light curve was $0.067 \pm 0.006(\sim 11 \sigma)$ and $0.069 \pm 0.006(\sim 11.5 \sigma)$ in 

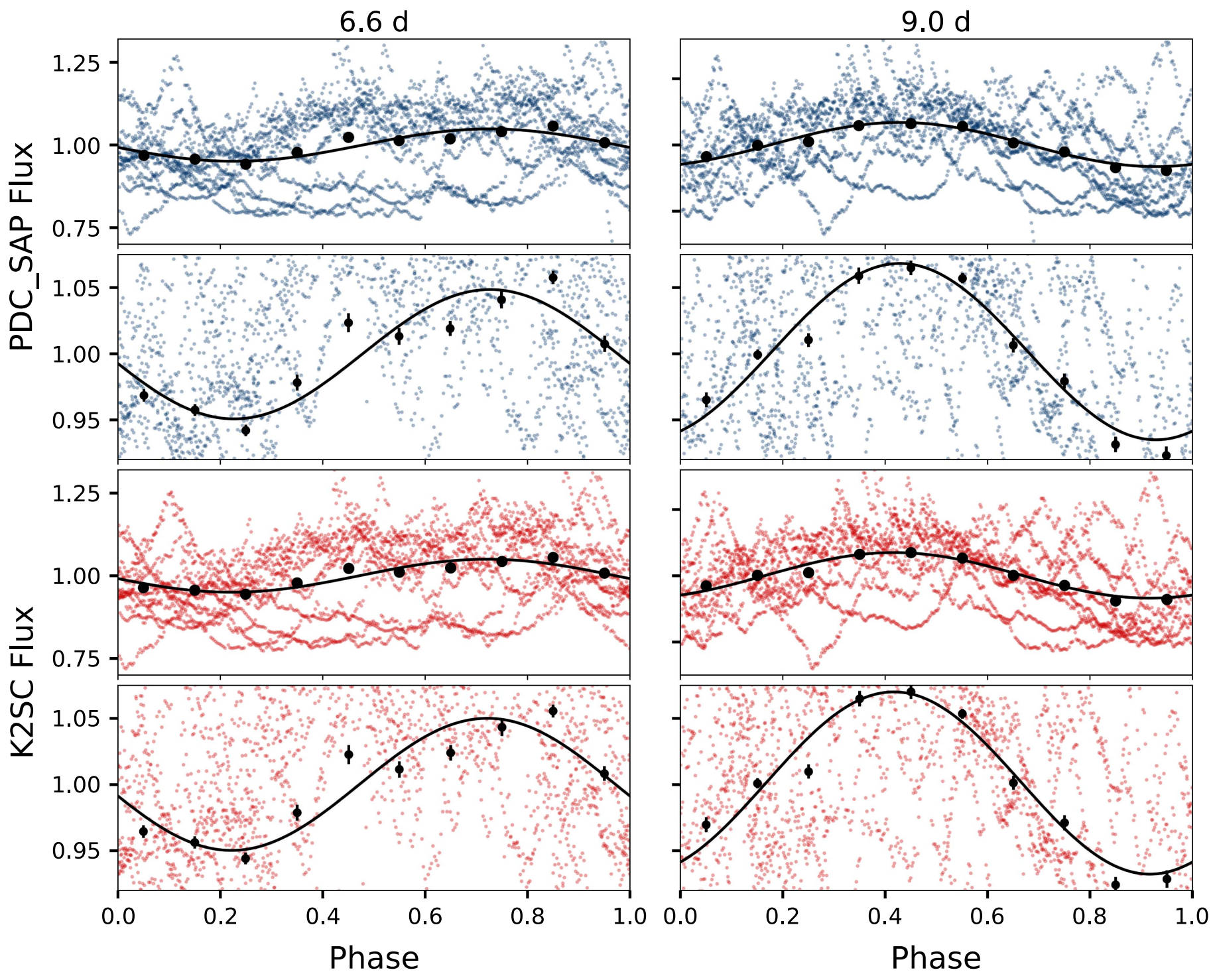

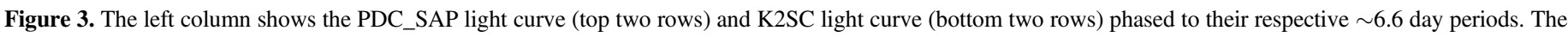

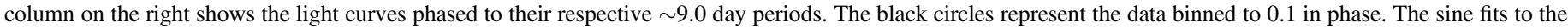
binned data are also displayed in black. The second and fourth rows scale the $y$-axis to the amplitude of the binned flux to aid the eye.

normalized flux, respectively. The amplitudes are large compared to their uncertainties, which further reinforces the significance of both signals.

In Figure 1, other peaks are apparent at 11.5 and 14.3 days; however, these peaks are not consistently evident when the data are examined in the two subsets illustrated in Figure 2. When considering only half the data, the number of periods of these potential signals that is covered is reduced. For the 11.5 day peak, 3.5 periods are covered, and for the 14.3 day peak, 2.8 periods are covered. However, for the 9 day peak, only 4.4 periods are covered: a $25 \%$ increase compared to the 11.5 day peak and a $50 \%$ increase on the 14.3 day peak. While this is relatively significant when considering the 14.3 day peak, it is fairly marginal for the 11.5 day peak, yet the 9 day peak survives strongly in all the periodograms of Figure 2. There is also a significant peak in the 20-27 day period range in the analysis of the full light curve and in the first and second half subsets. Given the $\sim 80$ day duration of the campaign, the full data set covers just over three full periods for the $\sim 24$ day signal. It is thus unlikely that there is a true periodicity at 24 days. When we phase the full data set to a 24 day period, the result does not suggest a sinusoidal like that seen in Figure 3. Therefore, at this time we are not convinced that the 24 day period is real. We also note that for a period of 6.6 and 9.0 days, the light curve should show a beat period at exactly 24.75 days. Future compilation of all groundbased observations may shed light on the potential sources of these signals.

We obtained a new estimate of the $v \sin i$ of CI Tau using the optical echelle spectra presented in Johns-Krull et al. (2016). In order to work with the highest possible signal-to-noise data available to us, we used the least squares deconvolution (LSD) profiles that were computed by Johns-Krull et al. (2016) giving us a total of 26 observations of CI Tau with which to measure the $v \sin i$. LSD (Donati et al. 1997) is a technique similar to cross-correlation and is used to compute an average line profile. In the case of our CI Tau spectra, a total of 1944 photospheric absorption lines were used to compute the average line which has a line-depth weighted mean wavelength of $6118 \AA$ A. In order to estimate the $v \sin i$ of CI Tau we computed the LSD profile, using the same 1944 lines, of HD 65277 observed on 2013 
November 15 with the same setup as the observations of CI Tau (Johns-Krull et al. 2016). HD 65277 is a K5V star with a $v \sin i \leqslant 0.53 \mathrm{~km} \mathrm{~s}^{-1}$ (Martínez-Arnáiz et al. 2010), and therefore represents an essentially non-rotating star of very similar spectral type to CI Tau. To measure the $v \sin i$ we then rotationally broadened the LSD profile of HD 65277, using a standard rotational broadening kernel (e.g., Gray 2008) with a limb-darkening coefficient of 0.72 , appropriate for the spectral type of CI Tau (K7) and the wavelength $(6118 \AA)$ of the line being analyzed. We broadened the HD 65277 LSD profile with $v \sin i$ values ranging from 1 to $20 \mathrm{~km} \mathrm{~s}^{-1}$ in steps of $1 \mathrm{~km} \mathrm{~s}^{-1}$ and measured the FWHM of the resulting broadened profile in order to define the relationship between $v \sin i$ and FWHM. We then measured the FWHM of each of the 26 CI Tau LSD profiles and interpolated on the defined relationship to determine the $v$ sin $i$ of CI Tau. Taking the mean and the standard deviation of the mean as the value of the random error, we find $v \sin i=10.08 \pm 0.14 \mathrm{~km} \mathrm{~s}^{-1}$ for CI Tau. In order to estimate a systematic error we first repeated the process using limb-darkening coefficients of 0.54 and 0.78 , which represent the two extremes of the coefficients based on the full wavelength range of the lines used to compute the LSD profile. Taking the largest difference with our original measurement, we assume this is the (likely over)estimate $\left(0.25 \mathrm{~km} \mathrm{~s}^{-1}\right)$ of the systematic error resulting from our choice of limb-darkening coefficient and rotational broadening kernel. We then attempted to estimate the error that might result from using the LSD profiles themselves. We rotationally broadened the full observed HD 65277 spectrum by $10 \mathrm{~km} \mathrm{~s}^{-1}$ and then computed the LSD profile of this broadened spectrum. We then measured the FWHM and used our same relationship to find its $v \sin i$. Taking the difference $\left(0.12 \mathrm{~km} \mathrm{~s}^{-1}\right)$ with the true value gives a measure of the systematic uncertainty caused by using the LSD method in the first place. We then add all three sources of uncertainty in quadrature to get a final measurement for the $v$ $\sin i$ of CI Tau of $10.08 \pm 0.31 \mathrm{~km} \mathrm{~s}^{-1}$.

\section{Discussion}

From the Lomb-Scargle periodograms shown in Figures 1 and 2 , it is clear that there are at least two significant astrophysical signals in the $K 2$ light curve of CI Tau at $\sim 6.6$ and $\sim 9.0$ days. We identify the $\sim 6.6$ day signal as the stellar rotation period. We use our $v \sin i$ measurement of CI Tau, and the inclination of CI Tau's disk, $i=45^{\circ} .7 \pm 1.1$ (Guilloteau et al. 2014), to estimate the stellar radius. Assuming the disk and stellar spin axes are aligned, we have

$$
R_{\star}=\frac{1.96 \times 10^{-2} \times P_{\mathrm{rot}}(\text { days }) \times v \sin i\left(\mathrm{~km} \mathrm{~s}^{-1}\right)}{\sin i}
$$

where $P_{\text {rot }}$ is the period from the Lomb-Scargle periodogram analysis. For the peak at $P=6.6$ days this corresponds to a stellar radius, $R_{\star}=1.81 \pm 0.08 R_{\odot}$, whereas the period at $P=9.0$ days would correspond to a stellar radius of $R_{\star}=2.50 \pm 0.15 R_{\odot}$. From the luminosity of CI Tau $\left(L_{\star}=0.81 L_{\odot}\right)$ and an effective temperature of $4060 \mathrm{~K}$ (McClure et al. 2013), we estimate the radius of the star from $L_{\star}=4 \pi R_{\star}^{2} \sigma T_{\text {eff }}^{4}$ to be $R_{\star}=1.81 R_{\odot}$, consistent with the $\sim 6.6$ day period but inconsistent with the $\sim 9.0$ day period. For a K7 star of age $\sim 2 \mathrm{Myr}$, a radius of $\sim 2.5 R_{\odot}$ is unrealistic (e.g., Baraffe et al. 2015).
With the identification of the $\sim 6.6$ day stellar rotation period, the question remains, what is origin of the $\sim 9.0$ day signal in the periodogram? Because of $K 2$ 's large pixel size, we considered the possibility of contamination from additional sources in the field and found that images from DSS, Galex, 2MASS, WISE, and PanSTARRS show that there are no objects of comparable brightness within $1^{\prime}$ of CI Tau. We also examined the potential for multiplicity in the system. Highresolution observations reaching $5 \sigma$ contrast at 0 ". 25 separation (Uyama et al. 2017) provide no evidence for a companion down to $\Delta H=6.8$ that could contribute to the photometric signal. However, there is evidence in support of a substellar body. Johns-Krull et al. (2016) reported the detection of a planet orbiting CI Tau using data from an extensive optical and infrared RV survey. The planet mass they derived is $M_{p}=11.29 \pm 2.16 \quad M_{\mathrm{Jup}}$ and the orbital period is $P_{\text {orb }}=8.9891 \pm 0.0202$ days, consistent with the $\sim 9.0$ day period shown in both of the Lomb-Scargle periodograms in Figure 1. Our current understanding of the CI Tau system is that the planet does not transit. This is supported by evidence that the disk is inclined $i \sim 45^{\circ}$ (Guilloteau et al. 2014), though it is possible that a planet could be in an orbit misaligned with disk mid-plane (e.g., Kepler-63; Sanchis-Ojeda et al. 2013). However, the $\sim 9.0$ day periodic signal may be the result of a planet-disk interaction because the presence of a massive planet in an actively accreting disk should show both spectroscopic and photometric variability. Indeed, Johns-Krull et al. (2016) find evidence in the $\mathrm{H} \alpha$ profile variations of CI Tau that the planet may be modulating the accretion of disk material onto the star. Although hotspots located at the foot of accretion streams can affect RV measurements mimicking the signal of an orbiting body (Kóspál et al. 2014; Sicilia-Aguilar et al. 2015), Johns-Krull et al. (2016) specifically looked for these signals and found no evidence that hotspots produced the $\mathrm{RV}$ signals seen in photospheric absorption lines. Given the stellar mass of $0.80 M_{\odot}$ (Guilloteau et al. 2014), Johns-Krull et al. (2016) determined a semimajor axis of $0.079 \mathrm{au}$ for the planetary orbit. This would place the planet inside the inner edge of the disk at $0.12 \mathrm{au}$ (McClure et al. 2013). It is probable that an $11.3 M_{\text {Jup }}$ planet so close to the inner edge of the disk would stimulate the accretion of material and possibly modify accretion onto the star, creating non-axisymmetric accretion flows (Tofflemire et al. 2017a, 2017b). As the planet orbits the star, this interaction could produce a periodic variation in the $\mathrm{H} \alpha$ emission, a tracer of accretion. The impact on stellar accretion could produce photometric variability on the $\sim 9.0$ day period of the planet's orbit, which $\mathrm{K} 2$ data show as a periodic component in the system brightness. Additionally, the strength of this periodic component can be expected to fluctuate because of the sporadic nature of accretion on short timescales (Herbst et al. 2007). This is observed in the analysis of the first and second halves of the data and is in contrast to the relatively consistent signal strength of the $\sim 6.6$ day periodic component in brightness, which is caused by cold starspots that are long-lived in young stars (e.g., Stelzer et al. 2003) and produce relatively consistent fluctuations in brightness as the star rotates (Herbst et al. 2007; Bradshaw \& Hartigan 2014). If the 9.0 day periodic signal is the result of stellar rotation, it would call into question the legitimacy of the planet. On the other hand, the similar RV amplitudes seen in the optical and the IR and the null results on tests for RV variations produced by an accretion hotspot (Johns-Krull et al. 2016) attest to the 
significance of planet's detection. In addition, we would then need to explain the source of the 6.6 day signal, which is the most persistent signal observed in Figures 1 and 2.

The exact mechanism by which a hot Jupiter might itself accrete and modulate disk accretion onto a young star has not been simulated to our knowledge. However, there have been several simulations of accretion from circumbinary disks onto the central stars in the binary system (Artymowicz \& Lubow 1996; Günther \& Kley 2002; Muñoz \& Lai 2016). Most of these simulations focus on equal-mass or nearly equal-mass stars in either circular or eccentric orbits. For equal-mass stars in circular orbits, quasi-periodic variations in the accretion rate are seen, but with a dominant period that is a few times the binary orbital period (e.g., Muñoz \& Lai 2016). For eccentric systems, the accretion is seen to vary with a period equal to the binary orbital period. Johns-Krull et al. (2016) find a best-fit solution to their RV measurements for an eccentric orbit of the planet around CI Tau; however, the uncertainty in the eccentricity is large enough that the orbit could be circular. The mass ratio found by Johns-Krull et al. (2016) for the CI Tau star and planet system is $\sim 0.014$. D'Orazio et al. (2013) simulate black hole binaries in circular orbits with a range of mass ratios from 1.0 down to 0.003 . At a mass ratio of 0.01 they find that visible accretion streams are still present with the stream corotating with the binary orbital period. Such a scenario would produce accretion modulated with a period equal to the binary orbit when viewed from a fixed location (e.g., the Earth). Furthermore, periodic accretion variability has been observed in eccentric $\mathrm{T}$ Tauri binaries (Tofflemire et al. 2017a, 2017b). While these scenarios are not identical, a young star plus (potentially eccentric) hot Jupiter just inside the disk truncation radius is consistent with observations of CI Tau, and these and similar simulations and observations suggest that the accretion onto the central star can show modulation of the flux or spectral features with the binary orbital period. We suggest this is the source of the $\sim 9.0$ day periodicity seen in the $\mathrm{K} 2$ data for CI Tau.

\section{Summary}

Our periodogram analysis of the $K 2$ photometry of CI Tau reveals the presence of two persistent periodic signals at $\sim 6.6$ and $\sim 9.0$ days. We find that the $\sim 6.6$ day signal likely corresponds to the stellar rotation period because in conjunction with our $v \sin i$ measurement, this value yields a reasonable estimate for the stellar radius, consistent with the effective temperature of a K7 star with CI Tau's luminosity (McClure et al. 2013). If the rotation period is 9.0 days, the radius becomes unphysically large. The $\sim 9.0$ day signal is consistent with the orbital period of a non-transiting, several Jupiter-mass planet located near the inner edge of the system's accretion disk (Johns-Krull et al. 2016). We postulate that the $\sim 9.0$ day signal originates from planet-disk interactions and the impact on the accretion of disk material onto the star is modulated by the planet's orbit and manifests in the system's photometric variability. The scenario by which a Jupiter-sized planet could influence accretion from a disk onto a star has not been simulated as far as we know. However, simulations of black hole binaries (D'Orazio et al. 2013) have shown that with a mass ratio comparable to $\mathrm{CI}$ Tau $\mathrm{A}$ and $\mathrm{b}$, it is possible that visible accretion streams can be regulated with a period equal to the orbit of the binary. Although this scenario does not simulate star-disk-planet interactions, it does suggest the possibility that a planet located within a disk's truncation radius can modulate accretion onto its host star with a period similar to its orbit. A model that simulates such a scenario, as is demonstrated in the CI Tau system, would supplement our understanding of the physical processes that occur as planets interact with disk accretion streams onto young stars.

We are grateful to S. Aigran, H. Parviainen, A. Vanderburg, and R. Luger for their valuable insight and assistance with $K 2$ detrending procedures. We thank Wei Chen for their contribution to the $v \sin i$ we report in this Letter. C.M.J.-K. would like to acknowledge useful discussions with P. Hartigan. L.I.B. wishes to thank L. Nofi for thoughtful discussion and insight. We would also like to extend our gratitude to the anonymous referee who provided valuable input that improved this work. This Letter includes data collected by the $K 2$ mission. Funding for the Kepler mission is provided by the NASA Science Mission directorate. All of the data presented in this Letter were obtained from the Mikulski Archive for Space Telescopes (MAST). STScI is operated by the Association of Universities for Research in Astronomy, Inc., under NASA contract NAS526555. Support for MAST for non-HST data is provided by the NASA Office of Space Science via grant NNX09AF08G and by other grants and contracts. This research has made use of NASA's Astrophysics Data System Bibliographic Services and the SIMBAD database, operated at CDS, Strasbourg, France.

\section{ORCID iDs}

Lauren I. Biddle (iD https://orcid.org/0000-0003-2646-3727 Joe Llama (i) https://orcid.org/0000-0003-4450-0368

\section{References}

Aigrain, S., Hodgkin, S. T., Irwin, M. J., Lewis, J. R., \& Roberts, S. J. 2015, MNRAS, 447, 2880

Aigrain, S., Parviainen, H., \& Pope, B. J. S. 2016, MNRAS, 459, 2408

Artymowicz, P., \& Lubow, S. H. 1996, ApJL, 467, L77

Baraffe, I., Homeier, D., Allard, F., \& Chabrier, G. 2015, A\&A, 577, A42

Borucki, W. J., Koch, D., Basri, G., et al. 2010, Sci, 327, 977

Borucki, W. J., Koch, D. G., Basri, G., et al. 2011, ApJ, 728, 117

Bouvier, J., Alencar, S. H. P., Harries, T. J., Johns-Krull, C. M., \& Romanova, M. M. 2007, in Protostars and Planets V, ed. B. Reipurth, D. Jewitt, \& K. Keil (Tucson, AZ: Univ. Arizona Press), 479

Bradshaw, S. J., \& Hartigan, P. 2014, ApJ, 795, 79

Burke, C. J., Bryson, S. T., Mullally, F., et al. 2014, ApJS, 210, 19

Cody, A. M., Stauffer, J., Baglin, A., et al. 2014, AJ, 147, 82

David, T. J., Hillenbrand, L. A., Petigura, E. A., et al. 2016, Natur, 534, 658

Donati, J. F., Moutou, C., Malo, L., et al. 2016, Natur, 534, 662

Donati, J.-F., Semel, M., Carter, B. D., Rees, D. E., \& Collier Cameron, A. 1997, MNRAS, 291, 658

D’Orazio, D. J., Haiman, Z., \& MacFadyen, A. 2013, MNRAS, 436, 2997

Gray, D. F. 2008, The Observation and Analysis of Stellar Photospheres (Cambridge: Cambridge Univ. Press)

Guilloteau, S., Simon, M., Piétu, V., et al. 2014, A\&A, 567, A117

Günther, R., \& Kley, W. 2002, A\&A, 387, 550

Herbst, W., Eislöffel, J., Mundt, R., \& Scholz, A. 2007, in Protostars and Planets V, ed. B. Reipurth, D. Jewitt, \& K. Keil (Tucson, AZ: Univ. Arizona Press), 297

Howard, A. W., Marcy, G. W., Johnson, J. A., et al. 2010, Sci, 330, 653

Howell, S. B., Sobeck, C., Haas, M., et al. 2014, PASP, 126, 398

Ivezić, Ž, Connelly, A. J., VanderPlas, J. T., \& Gray, A. 2014, Statistics, Data Mining, and Machine Learning in Astronomy (Princeton, NJ: Princeton Univ. Press)

Jenkins, J. M., Caldwell, D. A., Chandrasekaran, H., et al. 2010, ApJL, 713, L87

Johns-Krull, C. M., McLane, J. N., Prato, L., et al. 2016, ApJ, 826, 206

Kopparapu, R. K. 2013, ApJL, 767, L8 
Kóspál, Á., Mohler-Fischer, M., Sicilia-Aguilar, A., et al. 2014, A\&A, 561, A61 Luger, R., Agol, E., Kruse, E., et al. 2016, AJ, 152, 100

Luger, R., Kruse, E., Foreman-Mackey, D., Agol, E., \& Saunders, N. 2017, arXiv: 1702.05488

Mann, A. W., Newton, E. R., Rizzuto, A. C., et al. 2016, AJ, 152, 61

Martínez-Arnáiz, R., Maldonado, J., Montes, D., Eiroa, C., \& Montesinos, B. 2010, A\&A, 520, A79

McClure, M. K., Calvet, N., Espaillat, C., et al. 2013, ApJ, 769, 73

Muñoz, D. J., \& Lai, D. 2016, ApJ, 827, 43

Press, W. H., Teukolsky, S. A., Vetterling, W. T., \& Flannery, B. P. 1992, Numerical Recipes in C. The Art of Scientific Computing (2nd ed.; Cambridge: Cambridge Univ. Press)

Queloz, D., Henry, G. W., Sivan, J. P., et al. 2001, A\&A, 379, 279
Robertson, P., Mahadevan, S., Endl, M., \& Roy, A. 2014, Sci, 345, 440 Sanchis-Ojeda, R., Winn, J. N., Marcy, G. W., et al. 2013, ApJ, 775, 54 Sicilia-Aguilar, A., Fang, M., Roccatagliata, V., et al. 2015, A\&A, 580, A82 Stelzer, B., Fernández, M., Costa, V. M., et al. 2003, A\&A, 411, 517

Tofflemire, B. M., Mathieu, R. D., Ardila, D. R., et al. 2017a, ApJ, 835, 8 Tofflemire, B. M., Mathieu, R. D., Herczeg, G. J., Akeson, R. L., \& Ciardi, D. R. 2017b, ApJL, 842, L12

Uyama, T., Hashimoto, J., Kuzuhara, M., et al. 2017, AJ, 153, 106

Vanderburg, A., Montet, B. T., Johnson, J. A., et al. 2015, ApJ, 800, 59

VanderPlas, J. T. 2017, arXiv:1703.09824

Winn, J. N., \& Fabrycky, D. C. 2015, ARA\&A, 53, 409

Yu, L., Donati, J.-F., Hébrard, E. M., et al. 2017, MNRAS, 467, 1342

Zechmeister, M., \& Kürster, M. 2009, A\&A, 496, 577 